Relmecs, diciembre 2018, vol. 8, no. 1, e033, ISSN 1853-7863

Universidad Nacional de La Plata. Facultad de Humanidades y Ciencias de la Educación. Centro Interdisciplinario de Metodología de las Ciencias Sociales.

Red Latinoamericana de Metodología de las Ciencias Sociales

\title{
Otra controvertida relación maestro- discípulo. Pierre Bourdieu y Luc Boltanski, en torno al problema de la acción y la axiología del investigador
}

\author{
Another controversial teacher-disciple relationship. Pierre Bourdieu \\ \& Luc Boltanski, around the problem of the action and the axiology \\ of the researcher
}

\author{
Gabriel Nardacchione * y Pablo Tovillas * \\ * Universidad de Buenos Aires, Argentina | gabriel.nardacchione@gmail.com , tovillaspablo@hotmail.com
}

\section{PALABRAS CLAVE RESUMEN}

\begin{tabular}{|c|c|}
\hline $\begin{array}{r}\text { Estructural } \\
\text { Constructivismo } \\
\text { Sociología pragmática } \\
\text { Habitus } \\
\text { Prueba } \\
\text { Campo } \\
\text { Régimen de acción }\end{array}$ & $\begin{array}{l}\text { Este artículo busca medir las diferencias entre la sociología estructural constructivista de P. Bourdieu y la } \\
\text { sociología pragmática de L. Boltanski. Elegimos recorrer algunos ejes de las obras de ambos autores, } \\
\text { planteando en cada uno hipótesis de ruptura o de continuidad. Primero, la forma en que definen el problema } \\
\text { de la acción y cómo esta se genera. Segundo, los espacios en los que se desarrolla la acción y de qué manera } \\
\text { la condicionan. Y por último, cómo tratan el problema de la ética política y la dimensión axiológica del } \\
\text { trabajo del sociólogo. }\end{array}$ \\
\hline Ética política & \\
\hline KEYWORDS & ABSTRACT \\
\hline $\begin{array}{r}\text { Structural } \\
\text { Constructivism } \\
\text { Pragmatic sociology } \\
\text { Habitus } \\
\text { Test }\end{array}$ & $\begin{array}{l}\text { This article seeks to measure the differences between the structural constructivist sociology of P. Bourdieu } \\
\text { and the pragmatic sociology of L. Boltanski. We chose to cover some axes of the works of both authors, } \\
\text { proposing in each one hypothesis of rupture or continuity. First, the way they define the problem of the } \\
\text { action and how it is generated. Second, the spaces in which the action takes place and in what way they } \\
\text { condition it. And finally, how they deal with the problem of political-ethics and the axiological dimension of } \\
\text { the sociologist's work. }\end{array}$ \\
\hline
\end{tabular}

Action regime

Political ethics 


\section{Introducción}

Este artículo busca medir las diferencias entre la sociología estructural constructivista de P. Bourdieu y la sociología pragmática de L. Boltanski sobre algunos aspectos específicos de sus obras. Para ello, elegimos recorrer algunos ejes de ambas obras, planteando en cada uno una hipótesis de ruptura o de continuidad. Se considerará la producción de la obra de la escuela de P. Bourdieu desde los inicios de los años 60 del siglo pasado, momento del surgimiento del estructuralismo genético, hasta su consolidación a principios de 1980 (de allí que se tendrán en cuenta las obras de ese período); y el movimiento de la sociología pragmática puesto en marcha por L. Boltanski a comienzos de los 80 , como nacimiento de un proceso de diferenciación conceptual y metodológica que transitaremos hasta comienzos de los años 2000. Con este propósito, tomamos un momento de intersección entre ambas obras: los años 80, momento en que la obra de P. Bourdieu comienza a ser criticada por la aparición de la sociología pragmática.

El artículo está compuesto de tres partes. Cada una de ellas se relaciona con tres dimensiones de análisis de la obra de cada autor. Primero, la forma en que definen el problema de la acción y cómo ésta se genera. Segundo, los espacios sobre los que se desarrolla la acción y de qué manera la condicionan. Tercero, cómo tratan el problema de la ética política y la dimensión axiológica del trabajo del sociólogo. En la conclusión esbozaremos nuestras hipótesis de continuidad o de ruptura entre las obras de ambos autores sobre cada uno de los ejes mencionados.

\section{El problema de la acción}

\section{El habitus como concepto mediador}

Para el estructuralismo genético la acción social es producto del habitus, generador de prácticas y representaciones. La concepción de la acción remite a una concepción disposicional que se inserta en un sistema de conceptos: el habitus en relación con el espacio social estructurado, el campo, los capitales y el sentido práctico. P. Bourdieu presentó una primera formulación del concepto de habitus en Un arte medio (2003), pero será en Esquisse d'une theorie de la pratique (1972) y luego en Le sens pratique (1980) donde será expuesta de forma metódica, sistemática y detallada.

En las definiciones que P. Bourdieu da de habitus se yuxtaponen dos vertientes del concepto: la pasiva y la activa, la del antes y la del después. El primer componente, el de la socialización y el segundo, el de las prácticas (o acción) no es cronológico sino dialéctico: no hay un tiempo de interiorización de las disposiciones seguido de un tiempo para las prácticas, sino simultáneamente interiorización de las experiencias de exteriorización y externalización de lo interiorizado. Las acciones sociales son por lo general una expresión "no reflexiva" de disposiciones adquiridas tempranamente e inscriptas en forma de disposiciones y capacidades en los habitus (Lebaron y Mauger, 2012, p. 134).

La interiorización de la exterioridad se produce según P. Bourdieu por intermedio de dos tipos de procesos de socialización: los implícitos o por simple familiarización, y los explícitas o metódicos producidos por el sistema escolar fundamentalmente, pero también por instituciones religiosas, políticas, sindicales, etc. Estaríamos entonces ante un habitus primario producto de las inculcaciones iniciales y "llamadas al orden" que recibimos en nuestra primera infancia y que son, por tanto, las más antiguas y las más perdurables. Se trata, por ejemplo, del aprendizaje de maneras de caminar, de hablar, de comer, de gustos y de aversiones que se vuelven naturales e inconscientes y, por tanto, se hallan al abrigo de las explicaciones y de una voluntad deliberada de transformación. Esta temprana incorporación de sociedad hace olvidar su génesis. En tal sentido, es la familia el lugar por excelencia en que se transmite este habitus originario o segunda naturaleza, como la llama P. Bourdieu. Las nuevas experiencias a las que son sometidas los habitus individuales estarán fuertemente condicionadas por esos primeros esquemas de percepción y acción aprehendidos. En el transcurso de la vida social se irá incorporando un 
habitus secundario producido por otras agencias de socialización (tanto formales como informales) de inculcación de cultura. El habitus procederá de estructuración en estructuración, toda vez que el agente afronte experiencias nuevas a lo largo de su trayectoria social.

Ahora bien, la acción no se produce en un vacío social, sino en el marco de un espacio social estructurado en el que los individuos ocupan distintas posiciones y poseen de manera desigual diferentes tipos de capital. A una clase determinada de condiciones de existencia corresponde un habitus de clase que produce no sólo unos gustos (o sistemas de clasificación específicos), sino un sentido de las posibilidades y las imposibilidades sociales para el agente (ej., el acceso o no a una institución escolar, club social, barrio, consumos, etc.).

Por otra parte, la acción social se produce en un marco más específico que P. Bourdieu denominará campos sociales, los cuales poseen diversos grados de autonomía entre unos y otros producto de la diferenciación de la sociedad. Si bien volveremos sobre el concepto de campo, podemos adelantar que habitus y campo conforman una relación de condicionamiento y de conocimiento.

El habitus habilita también la exteriorización de la interioridad. Las prácticas para P. Bourdieu no se pueden deducir ni de las condiciones presentes ni de las condiciones pasadas, sino que son el producto de una relación dialéctica entre una situación y un habitus. Es en La Distinción donde propone el siguiente esquema: [(habitus) + $($ campo $)=$ práctica . Es necesario poner en relación las condiciones sociales en las cuales se produce el habitus y aquellas de la puesta en marcha (la situación). En el encuentro entre el habitus como sistema de estructuras cognitivas y evaluativas y la situación se genera la práctica. La noción de sentido práctico permite a nuestro autor pensar el ajuste espontáneo de la acción del agente en un campo. El agente actúa “como se debe”, hace "lo único que se puede hacer" (las alternativas están constreñidas) y produce anticipaciones correctas (la metáfora deportiva sigue funcionando aquí). Para P. Bourdieu la polaridad estructura-acción no puede conceptualizarse como dos nociones antagonistas, ya que los cambios y transformaciones se producen merced también a la existencia de estructuras.

Un aspecto a destacar es que estos esquemas internos son durables y transformables. Si bien estas disposiciones pueden sufrir modificaciones en el curso de nuestras experiencias sociales, las mismas tienden a resistirse al cambio, poseen su propia inercia, y marcan así una cierta continuidad en la vida individual (ej., la adquisición temprana de formas de disponer el cuerpo condicionará intervenciones posteriores). Estas restructuraciones de los habitus se producen en función de la trayectoria social de los agentes en la que se interioriza lo social a medida que se experimenta cualquier movimiento en el espacio social (ej., el ascenso o descenso social producto de la adquisición o pérdida de capitales en sus distintas formas). También son transferibles debido a que estas disposiciones adquiridas en el transcurso de nuestra experiencia práctica tienen efectos sobre distintos campos. De esta forma el habitus confiere a las prácticas una independencia relativa en relación a las determinaciones exteriores del presente inmediato. Así podemos mantener taxonomías para descifrar una situación externa que ya sufrió modificaciones. Asegura también la presencia activa del pasado más allá de las normas formales y explícitas del presente.

Pensando en términos de disposición, P. Bourdieu nos conduce a razonar de manera relacional: el exterior que se interioriza y el interior que se exterioriza ${ }^{1}$. El habitus hace al ser y a un ser situado en un mundo social diferenciado. El habitus se expresa a través de esquemas clasificatorios de ordenamiento del mundo social, que conforman un conocimiento sin concepto. Dichos esquemas (o taxonomías cognitivas) han sido producidos históricamente a lo largo de generaciones y se adquieren mediante la práctica, aunque no funcionan como representaciones explícitas sino que se encuentran en "estado práctico".

Otro elemento a considerar es que las estrategias son el resultado de los habitus. Efectivamente estos esquemas de conocimiento permiten hacer frente a situaciones imprevistas y renovadas, aunque se encuentran constreñidas por las condiciones pasadas, esto es, por cómo se construyeron soluciones en el pasado que generaron una "huella" de acción, por lo tanto tienden a reproducir la realidad externa de la que son producto (Bourdieu, 1972, p. 202). Estas 
estrategias se adquieren desde la infancia al participar en actividades sociales y se incorporan como sentido práctico o sentido del juego a lo largo de la existencia.

En síntesis, no hay entonces diferencias sustantivas entre las estructuras incorporadas en el cuerpo y en la mente, y las estructuras externas objetivadas en las cosas y en las instituciones. Son, en suma, dos formas de existencia de lo social que mantienen entre ellas una complicidad ontológica. En un artículo publicado en 1980, "Le mort saisit le vif. Les relations entre l'histoire réifiée et l'histoire incorporée” (Bourdieu, 1980b), resalta la necesidad de poner en relación la historia objetivada, reificada, y la historia incorporada en forma de esquemas internos de clasificación o la "subjetividad objetiva” u “objetividad de segundo orden”, contenida en los habitus $\underline{\text {. }}$

Con su conceptualización del habitus P. Bourdieu quería de algún modo rescatar el aspecto "activo" del conocimiento práctico. Su objetivo era imprimirle dinámica y conflicto a las estructuras externas; los individuos juegan e interaccionan en y con dichas estructuras. Este concepto restablece, en cierta forma, la capacidad de elaborar la realidad que los agentes tienen sin desconocer que esa realidad está a, su vez, elaborada socialmente. No se trata ya de un sujeto trascendente propio de la filosofía, sino de un cuerpo y una mente que han adquirido, por la experiencia social, los principios que les permiten organizar la propia realidad social $\underline{3}$.

\section{La dinámica de las pruebas}

La acción pensada en términos pragmáticos no responde a ninguno de los dos grandes ejes tradicionales de la sociología: no es fruto de una intención o motivación individual (o de agregación colectiva), ni es derivado de una causa estructural o de un imperativo social. Tanto la "filosofía de la conciencia", que sitúa la orientación de la acción en la mente de los sujetos, como la "filosofía de lo social”, que sitúa las acciones sociales como determinaciones de un orden general, estructuran de manera diferente el problema de la acción en relación a la perspectiva pragmática (Latour, 2008). Desde esta perspectiva, la acción es más que la intención mentada de un individuo (aun en interacción social), y es menos que una determinación general (sea a través de una relación funcional, una estructura socioeconómica o un imperativo moral). A nuestro entender, la sociología de P. Bourdieu es heredera de esta antinomia, pues, si bien intenta articular sus términos, su teoría recae dentro de una preeminencia de las estructuras cognitivas e institucionales sobre las capacidades cognitivas de los sujetos.

Sobre este aspecto, la sociología pragmática cambia los puntos de anclaje. Según este punto de vista, la acción se configura en situación ${ }^{4}$. Estrictamente debería hablarse de una díada acción-situación ${ }^{5}$. No se puede pensar la acción por fuera de la situación, ni la situación fuera de la acción. Por un lado, las situaciones son locales, aunque su encadenamiento estructure formas de coordinación social de mayor escala. Ellas son configuraciones dinámicas sobre las que se cristalizan los procesos de interacción social, y funcionan como insumo (nunca determinante) de los esquemas cognitivos y morales para la acción. Por otro lado, la acción es la que produce el ajuste y reajuste de las situaciones confirmando mediante mecanismos adaptativos ciertas configuraciones o produciendo transformaciones creativas sobre las mismas. Este proceso práctico de ajuste-reajuste es lo que L. Boltanski y L.Thévenot (1991) llamaron involucramiento a la hora de presentar sus regímenes de acción $\underline{6}$. Según ellos, la sociología debe poner el foco sobre el involucramiento de las personas a su entorno, porque allí se puede comprender el sentido de las acciones, su relación problemática o de adaptación.

Este proceso de ajuste y reajuste práctico, según L. Boltanski y L. Thévenot (1991), articula competencias personales y dispositivos objetivos. A través de sus competencias, los actores pueden criticar una situación injusta o defenderla a través de una justificación. La tarea de la crítica no es corriente, es trabajosa, pues hay que probar lo que se critica. Además, no siempre se logra modificar las situaciones denunciadas. Se trata de una actividad de categorización compleja que requiere, en principio, percibir una situación desajustada (lo cual conlleva cierto malestar moral o incluso daño físico), sabiendo evaluarla según algún orden normativo ligado a la situación concreta. La competencia crítica de las personas lleva al interrogante: ¿cómo debería haberse actuado en esta situación? Así, un malestar se transforma en indignación y ésta en crítica político-moral. Pero estos procesos críticos dependen también de la inscripción de ciertos dispositivos. Como normalmente los actores dan prueba de 
sus críticas, ellos apelan a dispositivos situados que muestran lo que está sucediendo en el mundo. Los dispositivos hacen funcionar un mundo que cumple los principios morales, ya que para ello fueron creados. En ese sentido, si los dispositivos funcionan correctamente y resultan legítimos, entonces son resistentes como para evitar toda crítica. Pero si por el contrario el dispositivo se debilita, tanto en su funcionamiento (provocando accidentes, errores o disfunciones) como en su base moral y política (desmoronando su legitimidad), habilita a un sinnúmero de denuncias en favor de una reconfiguración de la situación. Allí se gesta un nuevo dispositivo moral.

A esta altura es necesario introducir una categoría clave: la prueba ${ }^{7}$. Quizás la prueba sea, desde la perspectiva pragmática, la noción más cercana a lo que la sociología tradicional entiende como "acción”. El problema del sentido de la acción se traduce en el ajuste de la prueba. Y si la acción involucra cambios o está sujeta a cierta reproducción social, para la perspectiva pragmática la prueba obliga a tests recurrentes, lo cual muestra una relación incierta entre las personas y las cosas. Pero, ¿cómo funciona la prueba? Por un lado la prueba significa probarse como actor con ciertas capacidades críticas. Las competencias no son propiedades de los actores, sino que emergen cuando se expresan (ej., el carácter reformista de un sindicato no existe sino hasta que emprende acciones que transforman la realidad de sus trabajadores). Pero al mismo tiempo la prueba remite a la necesidad de probar algo, de dar prueba material de lo que los actores están criticando. Allí los actores se las tienen que ver con las referencias objetivas de la situación a ser probada (ej., reglamentos que no se están cumpliendo, estadísticas de errores profesionales, etc.). En suma, probar y ser probado es algo que atraviesa tanto a los sujetos como a los objetos (dispositivos). Según la perspectiva de L. Boltanski, los sujetos se prueban entre sí (es decir, compiten críticamente entre sí), a la vez que buscan dar pruebas objetivas de lo que ocurre.

Por último, L. Boltanski muestra la dinámica histórica de la prueba a través de sus distintos modos de

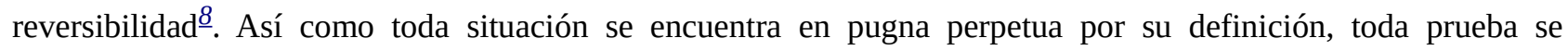
encuentra permanentemente sujeta a alguna redefinición crítica. L.Boltanski y E. Chiappelo (1999) muestran cómo el capitalismo "managerial" contemporáneo (tanto en sus principios como en sus dispositivos) se fue constituyendo a partir de dos tipos de críticas contra el capitalismo de masas: la crítica social y la crítica artística. Mientras la primera fue desdibujándose desde mediados del siglo XX, la segunda fue siendo incorporada a los dispositivos de poder. En otras palabras, las pruebas críticas en favor de autonomía y creatividad fueron siendo traducidas a dispositivos de orden que favorecieron la flexibilidad y la promoción de la creatividad en el ámbito laboral. Por ello, para tratar de manera simétrica esta dinámica histórica, L. Boltanski elaboró el formato bifronte de la prueba. Así, en una primera instancia, la prueba puede ser litigiosa $o$ de orden. Esto significa que una prueba puede constituirse tanto en crítica/denuncia como en defensa/justificación de un orden. L. Boltanski muestra cómo históricamente pruebas litigiosas han tenido éxito y han logrado cristalizarse en nuevos dispositivos (ej. la crítica socialista logró la negociación colectiva de salarios). Estos a su vez pueden volverse pruebas de orden frente a ulteriores pruebas litigiosas (ej., la socialdemocracia defiende la negociación colectiva frente a demandas liberales por su desregulación). Allí se expresa una dinámica típicamente pragmática: la de la crítica y la estabilización. En una segunda instancia, L. Boltanski y E. Chiapello (1999) dan cuenta de otros dos tipos de prueba: legítimas y de

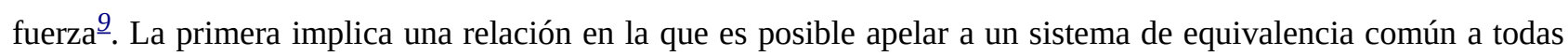
las personas. Dicho sistema puede ser funcional, legal o moral, pero, en cualquier caso, las relaciones entre las personas pasan "a través” de la codificación del sistema. La disputa en algún punto está mediada por esas referencias. Por el contrario, cuando no existen dichos equipamientos (dispositivos comunes) o las situaciones no se encuentran suficientemente categorizadas (o regladas normativamente), los actores se prueban (se miden) a través de la fuerza. Allí se manifiesta la violencia, la dominación de uno por otro, la sumisión, la amenaza, entre otras formas.

En síntesis, esta sociología de las pruebas introduce un dinamismo que potencia la incertidumbre de lo social $\underline{10}$. Así como los actores deben "saber pasar" de un formato de acción a otro sin que su manejo del entorno cambie sustancialmente, los dispositivos están permanentemente sujetos a pruebas y transformaciones. Contra varios supuestos de la sociología tradicional, desde esta perspectiva no hay posesión del poder (simbólico o material) que 
evite el desafío de la prueba. Todos tienen que dar pruebas de algo, los denunciantes y los defensores de un orden. La justificación de un orden, aun desde una posición de poder, implica siempre un despliegue de competencias para su configuración. En su análisis sobre el despliegue del capitalismo (Boltanski y Chiapello, 1999), los autores son claros acerca de cómo la organización del capitalismo requiere un trabajo sin pausa, no sólo a nivel material sino fundamentalmente a nivel de sus principios.

\section{El espacio de la acción}

\section{El campo como juego estructurado}

Con el concepto de espacio social estructurado, que es una manera de construir estructuras objetivas externas (la del campo de lucha de las clases), P. Bourdieu articulará otra manera de estructurar la realidad externa, que permitirá una mejor comprensión de zonas específicas y locales de la sociedad: la noción de campo en el que se desenvuelve la acción, que conforma un universo de prácticas relativamente autónomo. Así, el mundo social está compuesto por campos que son el resultado de un proceso de diferenciación producto de la división del trabajo social y que conduce a la existencia de campos autónomos. Estos microcosmos sociales en los que los agentes ocupan distintas posiciones poseen una autonomía relativa uno del otro ya que cada uno de ellos puede fijar reglas propias para protegerse de los influjos heteronómicos de otros campos sociales. El concepto de campo se encuentra asociado, como veremos, a otros conceptos ya elaborados por P. Bourdieu como habitus, violencia simbólica, capital, illusio y sentido práctico ${ }^{11}$.

La diferenciación que había sufrido la sociedad en sus diferentes dominios requería de una nueva herramienta. En sus análisis sobre Argelia había demostrado que, en un período de gran transformación social, las estructuras subjetivas quedaban desarraigas de las nuevas estructuras externas. Con la creación del concepto de campo podía pensar el cambio de las estructuras objetivas a través de la historia, la acción histórica de grupos dentro de esferas sociales más específicas. Esta idea permitía pensar no sólo la interacción entre grupos sociales sino también la organización de esos microcosmos.

La primera formulación de este concepto la encontramos en el artículo "Champ intellectuel et projet créateur" (Bourdieu, 1966, pp. 865-906). Aquí analiza la constitución del campo literario, su proceso de diferenciación a lo largo del siglo XIX a través de la formación de un propio mercado para sus obras, creación de sociedades de pensamiento, revistas, críticos, etcétera. Así, la elaboración de los juicios sobre la literatura no correspondía ya a las autoridades eclesiásticas, sino que se habían emancipado del poder de la Iglesia y se había conformado un universo autónomo con sus propias leyes y reglas de funcionamiento. Un campo puede definirse como una red o configuración de relaciones objetivas entre posiciones. La estructura de cada campo se corresponde con un estado de las relaciones de fuerza y de poder entre los agentes, los grupos y las instituciones que luchan por ocupar la posición hegemónica o la más poderosa.

A lo largo de los años 70 el concepto se fue desarrollando en la práctica investigativa. Los campos que fueron abordados son de densidad diversa, autonomía variable y diferente historia. La alta costura, la literatura y las artes, el mundo académico, el campo científico, el campo de la edición, el mercado inmobiliario, el campo de las historietas, el campo médico, el campo eclesiástico, el campo jurídico, el campo de las empresas, etc. son aprehendidos por medio de este cuadro general y retrabajados en cada caso a partir de material empírico $\frac{12}{2}$. Será tarea del sociólogo determinar para cada campo, y cada vez, cuáles son sus límites, qué especies de capital operan en él, cuáles son las posiciones de los que dominan y de los dominados y con qué mecanismos llevan adelante la dominación social, como así también revelar cuáles son las apuestas (lo que está en juego) de los jugadores en el contexto de cada campo/juego. Es decir, no es un acto de imposición del investigador sino un trabajo de inducción a través del análisis empírico caso por caso $\underline{13}$.

Dentro de cada campo los agentes cuentan con lo que P. Bourdieu denomina fe práctica, la cual es transmitida por 
los procesos de socialización y nos conduce a aceptar el mundo que nos circunda como evidente y natural. Por tanto, cada individuo es portador de una actitud dóxica, de una creencia, constituida de principios cognitivos y evaluativos que vuelven aceptable el mundo social que se expresa en cada campo. El papel del sociólogo será poner en evidencia, mediante los instrumentos de objetivación de su ciencia, esos principios indiscutidos o dóxicos que forman parte del "sentido común” de un campo (ej., quiénes son los dominantes, con qué principios dominan o qué capitales prevalecen como los más reconocidos).

P. Bourdieu establece una analogía entre campo y juego en la que los agentes, atrincherados en posiciones diferenciadas, establecen apuestas sobre la base de juegos estructurados. El juego se institucionaliza por la creencia de los agentes en él, no se ponen en cuestión sus reglas sino que se las reconoce. Existen regularidades y reglas explícitas e implícitas de funcionamiento. En determinados momentos, señala P. Bourdieu, los agentes portadores de habitus, que son a su tiempo transportables, luchan por subvertir esas mismas reglas que rigen la vida de un campo, y desarrollar estrategias heterodoxas. De esta forma, para el estructuralismo genético estos microcosmos sociales no conforman estructuras fijas o rígidas sino que son dinámicas y maleables. La toma de posición dentro de un campo será la dimensión activa de los agentes y los grupos sociales. Estas tomas de posición tienden a desestabilizar la situación para crear nuevas posiciones. Aquí resalta el aspecto de lucha política dentro de cada campo y, por tanto, una apertura a la indeterminación propia de un juego $\underline{\underline{14}}$.

¿Cómo se articula el concepto de habitus con el de campos sociales? El habitus y el campo conforman una relación de condicionamiento y de conocimiento. De condicionamiento ya que la pertenencia de un individuo a una posición en un campo social producirá un habitus, y de conocimiento porque la portación de un habitus contribuirá a significar (con su fe práctica) ese microespacio social (ej., los jugadores de un determinado deporte conocen las reglas por las que están a su vez condicionados). Bourdieu introduce aquí el concepto de interés: es lo que le importa al agente, lo que cuenta para él y lo que lo hace estar activo, salir de la ataraxia y de la indiferencia (Bourdieu, 2015, p. 317). El agente no ingresa a los campos sociales por una decisión deliberada y consciente sino que "nace en el juego y con el juego, y la relación de creencia, de illusio, de inversión es tanto más total, incondicional, cuanto se ignora como tal”. (Bourdieu, 2007, p. 108). El habitus nos permite evolucionar "con naturalidad" en un campo determinado sin tener que reflexionar sobre todas nuestras conductas y maneras de hacer: el habitus en cierta forma "economiza” el cálculo y la reflexión (Chistiane Chauviré \& Olivier Fontaine, 2008, pp. 68-69). Por último, es una relación de creencia práctica que afecta al cuerpo, el cual está habitado por esquemas motrices y automatismos adquiridos por la experiencia en el espacio social y en el campo específico. En un campo en el que la lógica de funcionamiento es diferente y los capitales en tanto recursos que se valorizan son distintos, el mismo agente puede no comprender las reglas de juego y, por tanto sentirse fuera.

Un aspecto a destacar en torno al habitus y su relación con los campos sociales es que existen desfases, discordancias y fallos en dicho vínculo, lo que nos lleva a pensar en el status de autonomía que pueden mantener los sistemas simbólicos para P. Bourdieu. Así, puede acontecer, sobre todo en momentos de profundas crisis sociales y, por tanto, de cambio en las condiciones sociales, que las disposiciones internas no estén adecuadas al campo y a los cambios en las posiciones sociales de los agentes en la distribución de los distintos capitales específicos. Los habitus, tienen entonces una inercia o una histéresis que hacen que sobrevivan a los cambios en las condiciones de producción de esos esquemas clasificatorios. Los individuos pueden adoptar posturas que se corresponden a una posición social anterior en el espacio social estructurado y en un campo (Bourdieu, 2006).

Por último, el concepto de sentido práctico: noción disposicional que articula los conceptos de campo y de habitus. A través de esta pareja de conceptos P. Bourdieu construye una forma de escapar del dilema de la espontaneidad personal y de la coerción social. El sentido práctico posibilita a los agentes, sin que estos tengan que realizar una reflexión "táctica” consciente, actuar inmediata y "naturalmente” en las situaciones sociales en las que se encuentran inmersos (lo que constituye un conocimiento sin concepto) $\frac{15}{15}$. Si relacionamos habitus, sentido práctico y campo podríamos decir que los agentes despliegan estrategias (ortodoxas y heterodoxas) que pueden cuestionar y cambiar la distribución de los poderes que otorgan los capitales en un campo social -es decir, 
las propias reglas que regulan la lógica de funcionamiento del campo-, o que puedan mantener el orden y por tanto desarrollar acciones ortodoxas.

\section{Acción situacional y pluralismo gramatical}

Durante el siglo XX la sociología fue desarrollando un interés por comprender la acción y particularmente por estudiar los ámbitos en donde se inscribe la misma. A nuestro entender, este es un proceso que va desde una acción social, entendida dentro de un marco social uniforme, hacia una creciente regionalización de la acción. Este proceso de regionalización va del comprensivismo weberiano a la influencia de la fenomenología, dentro de la sociología, de la mano de A. Schutz (1987). En otras palabras, para entender la acción de los sujetos era necesario no sólo liberarlos de ciertas estructuras, sino contextualizar sus prácticas. En ese sentido, la sociología bourdieusiana, a través del concepto de campo, da respuesta a esa inquietud por ciertas reglas de juego que dominan un conjunto de prácticas situadas.

En esta línea de análisis, la perspectiva pragmática intenta localizar aún más los ámbitos de acción, desechando la idea de campos homólogos (mercantil, administrativo, cultural, etc.) que atraviesan la sociedad. Las situaciones de acción no se encuentran configuradas sistemáticamente por un campo, ni condicionadas por su lógica de acción. La configuración situacional se aleja del isomorfismo bourdieusiano, que en cierta medida ancla un espacio de acción (corporación económica, burocracia municipal, ministerio de cultura, etc.) a la lógica de un campo. Desde la perspectiva pragmática, las situaciones se configuran de manera más flexible. El proceso de configuración es en sí una disputa de lógicas entre distintos ámbitos. Y aunque cada situación pueda encontrarse relativamente configurada, está al mismo tiempo abierta a su reconfiguración (Goffman, 2006; Becker, 2008). En ese marco de relativa incertidumbre y reversibilidad de la definición de las situaciones (Thomas, 2006), los actores deben “saberse adaptar” a múltiples variantes.

Para analizar dichos comportamientos situados, L. Boltanski (entre otros sociólogos pragmáticos $\frac{16}{}$ ) introdujo la

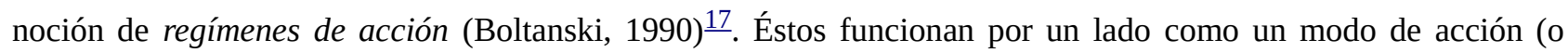
cuerpo de reglas más o menos tácitas) que expresa una forma de relacionarse entre personas en el mundo. Por otro lado, esta cosmovisión implica relaciones con las cosas, por ende, se ligan a los equipamientos materiales de las situaciones referidas. Allí aparecen los dispositivos que favorecen (o desfavorecen) que un régimen esté más o menos disponible en la situación (ej., el alquiler de un departamento se encuentra altamente codificado, a través de formas legales, formulas retóricas, etc., sin embargo, en algún caso ciertas personas prefieren resolver dicho acuerdo de palabra, apelando a la relación familiar que los une). En otros términos, las situaciones siempre favorecen determinadas gramáticas y desfavorecen otras. Pero, en cada caso, son los actores los que fuerzan el ajuste de una gramática (u otra) a la situación. Muchas veces lo hacen actuando en contra de situaciones equipadas, es decir, intentan cambiar los dispositivos situados.

Estos esquemas gramaticales, a los que L. Boltanski y todo su linaje académico hacen referencia, son formatos plurales que no piden exclusividad. Existen muchos, compiten entre sí y según cada situación se pueden activar uno u otro. Es decir, guardan distancia en relación con las situaciones a ser definidas. No se ligan necesariamente a ellas, pues al mismo tiempo pueden funcionar como matrices ordenadoras del mundo para muchas otras (ej., una negociación mercantil siempre va a estar marcada por una lógica de intercambio de bienes, pero eso no significa que no se deje de influir por algún acuerdo político entre sus jefes o por la notabilidad de un experto que recomienda un tipo de acuerdo). L. Boltanski y L. Thévenot (1999) llaman transporte de legitimidades a esta tarea de utilización de gramáticas ajenas a la definición tradicional de situación. En un estudio empírico, L. Boltanski (1990) muestra numerosos casos de denunciadores públicos que utilizan (hasta el abuso $\underline{18}$ ) legitimidades ajenas a la situación. Por ejemplo, reclamos locales sostenidos por sellos políticos o por alguna persona notable. Ambas legitimidades diferentes a las gramáticas del reclamo territorial. Este esquema de transportes muestra hasta qué punto las gramáticas no deben asociarse directamente a las situaciones, sino que dependen de las competencias de los actores para volver legítima una gramática a la situación. 
En el marco de esta pluralidad de regímenes y dada la dinámica temporal de ajuste a diferentes situaciones, los actores se encuentran obligados en muchos casos a realizar "pasajes" entre regímenes de acción (Dodier, 1991). En este caso su capacidad no pasa por redefinir la situación, sino por adaptarse a diferentes y sucesivos modos de acción. Puede tratarse tanto de situaciones diversas como de cambios en el tiempo de una misma situación. En cualquier caso, los cambios de regímenes ubican a los actores frente a la prueba de actuar con lógicas distintas, teniendo que adaptarse, para darle "continuidad” a la acción (ej., una movilización de un grupo de organizaciones de la sociedad civil por una demanda ambiental, en un momento debe atravesar una prueba político-legislativa, la cual puede permitirle resolver el caso a través de la redacción de una ley). Todo el tiempo, los actores se encuentran frente a diversas pruebas frente a las cuales deben saber actuar con naturalidad. En cualquiera de ellas, el primer punto pasa por saber actuar de la manera más eficaz bajo la lógica de la situación. En L'Amour et la justice comme compétences, L. Boltanski (1990) presenta los pasajes de un régimen a otro: entre la justicia, el amor, la rutina y la violencia (ver detalle en nota 43). Allí muestra los cambios gramaticales que realizan los actores en sus actividades ordinarias (ej., un representante de empresa de software negocia salvajemente los términos de un contrato comercial, pero a la hora de presentar el producto lo describe como un experto). Ese puntapié inicial va a dar lugar a un trabajo empírico de la sociología pragmática sobre las tareas que tienen que realizar los actores para poder pasar de un régimen al otro. En ese compendio de investigaciones (Boltanski \& Thévenot, 1989) $\frac{19}{\underline{19}}$ primaron los estudios sobre el pasaje de la rutina a la justicia (y viceversa). Es decir, cómo los actores logran salir de un modo de acción serial, donde se tiende a no reflexionar o a no discutir el funcionamiento de los dispositivos, para armar una causa en torno a alguna injusticia cometida. E inversamente, cómo los actores no logran construir dichas causas o, en todo caso, en qué medida necesitan de elementos de prueba de dicho mundo serial (funcionamiento de los dispositivos) para intentar crearlas.

Por último, L. Boltanski y L. Thévenot (1991) ya venían preocupados por el problema de los pasajes, pero, en este caso, dentro de un mismo régimen: el de la justicia. Allí, los autores desplegaron esquemas de justificación plurales que operan con una lógica similar a la de los regímenes, aunque con exigencias diferentes. Se trata de seis esquemas de referencias normativas que tienden a usarse como justificación de las propias acciones $\underline{20}$. Los seis órdenes de justicia funcionan como sistemas de equivalencia que todos reconocemos como universales (y que, por ende, en principio nos atenemos a ellos), aunque no todos los valoremos por igual. Menos acuerdo existe al momento de vincular alguno de ellos con alguna situación problemática específica que desencadena un debate de justicia. Allí, a menudo distintos grupos de actores hacen referencia a órdenes de justicia diferentes (ej., frente a la crisis de una empresa, un grupo de personas puede apelar al orden mercantil pidiendo la reconsideración de los contratos comerciales; otro grupo puede apelar al orden industrial pidiendo una planificación más estricta de los costos de la empresa, y finalmente otro grupo apelando al orden cívico puede pedir que se revea la reducción de horas trabajo con el fin de defender el salario de los operarios). Como vemos, los órdenes de justificación no se adhieren a determinados actores o situaciones, sino que en cada caso pueden ser movilizados por cualquier actor y también se encuentran relativamente disponibles dentro del equipamiento material (dispositivos) que ofrece cada situación. Esto significa que todas las personas pueden desplegar capacidades críticas, que éstas son plurales y que toda situación ofrece "pistas objetivas" (dispositivos) que sirven para desarrollar un argumento crítico. Sobre este último punto, L. Boltanski y L. Thévenot (1999) subrayan que un debate público tiene carnadura en el mundo. En ese sentido, muestran que los órdenes de justicia no son solamente criterios éticos y argumentos racionales, sino que se inscriben en el mundo real a través de dispositivos materiales en donde se anclan dichas ideas. En suma, las discusiones plurales remiten a ideas de bien común, pero fundamentalmente apuntan defender (o rechazar) dispositivos que se encarnan (o deberían encarnarse) en el mundo (ej., un grupo de desocupados no va a limitarse a legitimar su demanda o a ser reconocido por algún responsable político, sino a pedir que dicho reconocimiento llegue a un acuerdo -incluso una ley-- que "garantice" el cumplimiento de las respuestas a los reclamos). Incluso los pasajes que los actores realizan de un orden de justicia a otro siempre tienen en cuenta los dispositivos que se asocian a un principio o a otro (las demandas cívicas tienden a pedir leyes, las industriales: estadísticas, las domésticas: pruebas de confiabilidad, las mercantiles: balances superavitarios, y las de la opinión: índices de reconocimiento). 


\section{La axiología del investigador y la ética política}

\section{La sociología debe molestar}

Las investigaciones llevadas adelante por P. Bourdieu y sus discípulos han perseguido el objetivo de revelar cosas ocultas o reprimidas socialmente. Entre otras cuestiones han demostrado la correlación entre el léxico de la escuela (identificado con la "inteligencia") y el origen social (asociado al capital cultural heredado de la familia) (Bourdieu, 1990, p. 80). Otro ejemplo consiste en revelar que, desde el lado del público, la obra de arte requiere de instrumentos de conocimiento que no se hallan universalmente distribuidos entre las clases sociales y que tanto la obra de arte que realiza el artista como la capacidad del receptor para percibir se encuentran asociadas a un contexto social e histórico en el que están inmersos artista y público.

Para esto, el sociólogo deberá desarrollar un lenguaje analítico bajo una vigilancia epistemológica que se distinga del conocimiento del sentido común. P. Bourdieu propondrá una concepción integral del conocimiento del mundo social, que recupera la conexión entre el conocimiento teórico y el conocimiento práctico, entre la episteme y la doxa, entre dos conceptos del análisis social hasta ese momento escindidos en la tradición sociológica. Por una parte, es necesario sortear el realismo y la hipóstasis que el conocimiento objetivista (una forma de saber docto) hace de la estructura y de las relaciones objetivas, expulsando al individuo y al grupo por fuera de la historia, pero al mismo tiempo sin caer en un subjetivismo, cuya matriz no puede explicar la necesidad del mundo social. La teoría bourdieusiana se constituye entonces también como un rechazo al subjetivismo (como otro modo de conocimiento docto). Esta perspectiva subestima el poder de las estructuras objetivas externas a los individuos, su capacidad de influir y moldear las conciencias de los individuos. Ambos momentos, el objetivista y el subjetivista, resultan necesarios. Conforman una separación analítica pero no ontológica. Son dos dimensiones constitutivas de todo proceso social pero sin tener entre un momento y otro una relación de anterioridad ni de causalidad directas (Bourdieu, 2015, p. 229).

En este sentido, el lenguaje del sociólogo está compuesto por las hipótesis e instrumentos que el científico social construye al tiempo las que controla y reconfigura en su trabajo de investigación. En el caso de la sociología, dicha construcción consciente se impone con mayor fuerza, toda vez que el mundo social se autoconstruye en forma permanente. Al nombrar y clasificar lo social, el investigador no tiene nada de neutro, sino que se encuentra él mismo sumergido en un universo de relaciones sociales, lo que incluye su posición en un campo científico. Las clasificaciones producidas por el científico social (en los instrumentos estadísticos, en las definiciones institucionales, en una encuesta) es un acto que contiene una dimensión política y constituye una forma de modelar la sociedad. Por esta razón, el sociólogo debe controlar su práctica, lo que ve y lo que no ve, los objetos que elige. Para P. Bourdieu la sociología del conocimiento sociológico es una de las condiciones de la sociología científica. La objetividad es una conquista permanente que se encuentra bajo amenaza constante por la cultura de su tiempo.

Por otra parte, dirá P. Bourdieu, “debemos resistir a las palabras. El sociólogo no debe dejarse atrapar por categorías producidas por la sociedad para no ser hablado por aquellas clasificaciones producidas por grupos sociales e instituciones que por poseer legitimidad social se colocan fuera del juicio crítico”르. El conocimiento de lo social conforma entonces un arma contra la dominación cultural. Agentes dotados con mayores capacidades analíticas de su situación podrán adquirir un margen más amplio de libertad respecto a las necesidades que lo determinan. Estas armas deben ser científicas si quieren ser eficaces, ya que toda proposición que contradiga el sentido común se expone a la sospecha de ser partidista o ideológica y, por tanto, ser impugnada, no considerada en la esfera pública. Por eso la legitimidad de la ciencia en las sociedades contemporáneas exige a la sociología la utilización de teorías y métodos controlados y validados. El sociólogo debe ser constatativo para contar con una mayor fortaleza para enfrentar y confrontar con el discurso social performativo, cargado de deseos, exhortaciones, reproches, órdenes.

Asimismo, al tratar el sociólogo realidades materiales y simbólicas que implican relaciones de poder y dominación, "molesta" sacando a la luz realidades que ciertos grupos o individuos prefieren esconder o 
esconderse porque ellas perturban sus convicciones o intereses (Bourdieu, 1997). El mundo social para el autor no es un orden necesario e inmutable, aunque plantea que no se puede eludir el hecho de que existe un "orden" que se les impone a los agentes. El conocimiento de las leyes implícitas que constriñen las acciones sociales puede conducir a superar el orden arbitrario del mundo social y contribuir así a construir uno con otra racionalidad. La sociología de P. Bourdieu es una sociología de los condicionamientos. Pese a que los agentes no procesan como tales las coacciones que les impone el mundo social, dado que las desconocen, esto no quiere decir que no existan.

Por último, el sociólogo debe distinguirse de lo que P. Bourdieu llama un ingeniero social, el cual tiene por función proporcionar recetas y discursos performativos a los dirigentes de empresas o a los gobiernos. A contracorriente, el sociólogo debe comprender por comprender, y en ese acto ya cumple una función social, en virtud de que el poder es eficaz, en gran parte, por el desconocimiento de los mecanismos que lo legitiman, por las creencias o la doxa que fundamenta las relaciones de dominación.

\section{La emancipación es de los actores}

La impronta marxista dejó como legado una carga axiológica fundamental dentro de la sociología: el rol crítico del investigador. Desde antaño, y más allá de las diferencias teóricas o epistemológicas, el sociólogo debía “develar” los mecanismos ocultos, por los cuales se desenvolvía la dominación. Una mezcla de antropología negativa y holismo social llevaba a que dicha tarea consistiera en analizar las relaciones sociales de modo de explicar los factores más o menos complejos que hacían a la sociedad injusta. Heredero heterodoxo de esta tradición, el bourdieusianismo critica muchas figuras de la tradición marxista, pero recupera en lo central lo que tradicionalmente se adscribe a una "teoría de la sospecha": a) la sociedad no muestra lo que verdaderamente es, ni con qué patrones funciona, y b) sus actores desarrollan una "falsa conciencia"; carecen de reflexividad crítica como para invertir dichos patrones de injusticia. En ese cruce aparece la necesidad del sociólogo crítico.

Heredera de la tradición pragmatista, la sociología pragmática francesa critica estos dos supuestos de la sociología tradicional: a) que las relaciones sociales se explican por fuera de ellas mismas, como un mecanismo a develar a través de la racionalidad del investigador, y b) que los actores son básicamente no-conscientes de las relaciones en las que participan.

Frente al primer postulado, la sociología pragmática plantea que el distanciamiento crítico del investigador no le permite acceder a los mecanismos subyacentes de las relaciones sociales. Por el contrario, resulta un problema metodológico, pues esta sinopsis que realiza el sociólogo, en su afán crítico, produce un reduccionismo de lo social (Latour, 2001). En un sentido pragmatista, aleja la verdad de la realidad poniendo la verdad del investigador por sobre la "verdad pragmática” de los discursos y acciones en situación. Según esta perspectiva $\underline{22}$, la verdad no es una lógica oculta sino una práctica que logra ser eficiente en sus consecuencias, es decir, que encadena acciones. Para L. Boltanski, la axiología relevante es la del orden normativo de la situación $\underline{23}$. Por ello, la tarea del sociólogo consiste en describirlo, conocer sus reglas, sus márgenes de tolerancia. El análisis se centra en los ajustes y desajustes de los actores en relación con dicho orden. El sentido de la relación no surge de un saber externo, ni fundado en estructuras de conocimiento generales, sino a través de los intercambios concretos dentro de la situación. Así, la función crítica sólo puede estar enarbolada por enunciados de los actores que inciden sobre las formas categoriales con que se define la situación. Toda transformación será necesariamente producto de algún actor involucrado en la misma (por necesidad, por interés o por convicción). En suma, esta perspectiva desdibuja el rol crítico del investigador y lo desplaza hacia la capacidad crítica de los actores $\underline{24}$.

Frente al segundo postulado, esta perspectiva trata de "hacer hablar" a los mismos, poniendo en valor sus competencias críticas $\underline{25}$. El quid es metodológico e implica poner el foco sobre la voz de los actores, aunque su actividad no llegue a ser necesariamente crítica $\underline{26}$. Los actores siempre actúan siguiendo razones (más o menos conscientes) en toda situación en la que estuvieren involucrados. Pero este tipo de acción reconoce varias capas, o tiene distintos grados de reflexividad (Barthe et al, 2013, p. 188). En principio, saber actuar con naturalidad. Esto implica un conocimiento práctico sujeto a éxitos y fracasos. Saber actuar lo que las reglas de la situación exigen 
no va de suyo, sino que involucra un saber. En segunda instancia, los actores desarrollan competencias críticas, y cuestionan los límites que imponen las reglas. En estos casos, los actores desplazan marcos de interpretación, denuncian injusticias, construyen víctimas, entre otros mecanismos. Este recorrido está lleno de errores, retrocesos y aprendizajes, pues los actores no nacen con competencias críticas. Su tarea es reflexiva; aprenden a abstraerse de lo que ocurre en la situación para poder evaluarla y criticarla. Sólo así denuncian desajustes o injusticias y apelan a órdenes normativos que no se están cumpliendo en la situación. Recién en una tercera instancia puede ocurrir que estos mismos actores logren alguna transformación de los modos de relación entre las personas y las cosas, es decir, que no sólo denuncien alguna injusticia sino que logren modificar los soportes materiales de la situación (sus dispositivos). En resumen, sólo a través del análisis de la situación pragmática se pueden explorar los cambios de las relaciones entre las personas y las cosas를. Estos pueden ser inocuos, regresivos o progresivos (según el juicio de un observador externo), pero en cualquier caso son los que sin duda ocurren.

Para cerrar, sin duda que la faz axiológica de este giro metodológico se orienta a valorizar las capacidades críticas de los actores situados respetando el orden normativo de la situación y oyendo las capacidades críticas de sus actores. Si dicho giro tenía un fundamento epistemológico (el paso del saber experto al saber práctico), el momento axiológico del investigador consistiría en reforzar y amplificar las capacidades público-críticas de los actores situados $\underline{28}$. En algún sentido, intenta "simetrizar sus capacidades” para emprender operaciones críticas. Dar visibilidad a múltiples colectivos de reivindicación crítica implica fomentar la actividad democrática. El investigador es un ciudadano calificado que reflexiona sobre las prácticas sociales, por lo cual sus intervenciones producen consecuencias sobre las prácticas sociales ordinarias (Dewey, 2004). Pero, en este caso, las competencias de los actores son el punto de partida de la crítica y no el punto de llegada de la crítica de un saber sociológico. En estos términos, el apoyo reflexivo del investigador intenta reforzar las competencias críticas de los actores, las multiplica, les hace alcanzar escalas de acción a las cuales desde sus enunciados no podrían acceder. Les permite conectar con una multiplicidad de otros actores, que en otros casos desconocerían, y fundamentalmente les permite dinamizar el proceso de aprendizaje práctico. Pues su propio ensayo-error resulta más distinguible a través de la mirada de los investigadores que funcionan como caja de resonancia de los colectivos de reivindicación. Permanentemente, múltiples pruebas críticas se están desarrollando en distintos escenarios: en ámbitos locales o territoriales, dentro de los medios de comunicación, en el interior de las fábricas, en defensa del medio ambiente. Las así llamadas "incorrecciones gramaticales", por las cuales muchas críticas caen en desgracia, tienen que ver con la incompetencia de los actores para visualizar recursos, para categorizar correctamente, para adherir aliados, etc. Esta tarea de promoción, de despliegue de competencias busca reforzar sus capacidades reflexivas, al tiempo que recorre el sinuoso camino de la crítica.

\section{Conclusiones}

El análisis del problema de la acción social muestra cierta continuidad (aunque radicalizada) de la sociología pragmática de L. Boltanski frente al estructuralismo genético de P. Bourdieu. Lo que cambia el primero es el foco de atención sobre uno de los polos planteados por el paradigma bourdieusiano. Si P. Bourdieu buscaba articular estructura y acción, la sociología pragmática de L. Boltanski va a poner el acento sobre la acción en vínculo con la situación. Esto lleva a acentuar cierta indeterminación de la acción social por encima del peso de ciertas estructuras que condicionan repetidamente las prácticas sociales.

Fundamentalmente sobre los conceptos de habitus y sentido práctico, P. Bourdieu trata de articular el sentido de las acciones presentes en su vínculo con ciertas estructuras y/o eventos sucedidos en el pasado. Existe una codefinición entre la estructura y la acción; el sentido de lo actuado viene condicionado por un sinnúmero de patrones ya codificados. Los cambios son posibles, pero en el marco de dichas estructuras que limitan.

Por su parte, L. Boltanski va a poner el foco sobre las situaciones de la acción, sobre su presente y sobre cómo ellas se van definiendo sucesivamente. Se plantea una suerte de encadenamiento de situaciones que, en el marco de una definición de sentido, son pasibles de ser redefinidas por los actores. Esto introduce una dosis de 
incertidumbre de la acción que remite al concepto de prueba. La prueba pragmática es aquella que los actores deben enfrentar permanentemente en su curso de acción. No hay relación social sino a través de pruebas en las que los actores (y las cosas) se miden mutuamente. A una prueba $\mathrm{X}$ le sigue una prueba $\mathrm{X}$ ', y si bien la primera incide sobre la segunda, en ésta puede producirse un cambio (por mínimo que sea). L. Boltanski llama a esto reversibilidad de las pruebas. Dicho en jerga de la sociología clásica, la acción no se detiene nunca en un sentido, sino que debe renovarse permanentemente a través de una serie de prácticas. Aun cuando una acción aparezca como repetitiva, esto tiene más que ver con las prácticas de los actores implicados que con la determinación de una estructura externa.

Igual continuidad (aunque radicalizada) observamos sobre el análisis del espacio social donde se desarrolla la acción. En este caso, la sociología pragmática pretende hacer predominar un enfoque más dinámico. Esto significa que frente a cierta definición "topológica” de los campos de P. Bourdieu, aquélla refuerza el carácter problemático de las situaciones de acción, así como cierta pluralidad en los recursos gramaticales con los que dichas situaciones pueden definirse.

La sociología de P. Bourdieu define la noción de campo tanto para dar cuenta de un número variable de reglas que son legítimas en el interior del mismo, como para analizar de qué modo éstas se imponen sobre las prácticas. Esto implica la existencia de fronteras entre campos con algún grado de impermeabilidad aunque no herméticas. Es decir, la lógica de campo se impone en el interior del mismo y tiene dificultades para trasladarse.

Frente a esta perspectiva, la sociología pragmática de L. Boltanski pone de relieve la mutua definición entre la situación y un conjunto de gramáticas normativas. Este trabajo llevado adelante por los actores es en sí problemático e incierto. Si bien cada situación viene definida por un conjunto de gramáticas, no puede atribuírsele propiedad sobre ninguna de ellas. En cada situación (aun potencialmente), los actores pueden hacer intervenir distintas gramáticas (plurales aunque no infinitas) para definir las situaciones desde su perspectiva. Esta tarea de redefinición es permanente. No hay una regla normativa que se impone per se sobre una serie de prácticas, sino un conjunto heterogéneo de propuestas normativas que configuran polémica y sucesivamente el sentido de lo actuado.

No obstante, debe reconocerse que existe un conjunto de prácticas que desafían el carácter unívoco del sentido de las reglas -lo que fortalece la hipótesis de continuidad entre ambos paradigmas-. Los transportes de legitimidad, tan recurrentes y efectivos en la sociología pragmática, se expresan en algún sentido en las estrategias heterodoxas de los actores dentro de un campo. Allí, es necesario verificar cómo los actores logran trasladar la lógica de un campo extranjero a su propio campo de acción.

Por último, en relación a la axiología del investigador y a la incidencia de una ética política, ambos paradigmas también se diferencian en el marco de ciertas continuidades. El rol denunciador de la sociología de P. Bourdieu muta en un rol de promotor público de la sociología pragmática de L. Boltanski. En ambos casos hay una intención de crítica social, pero se diferencian en cómo entienden dicho trabajo.

En la sociología bourdieusiana la función crítica del investigador se basa en la denuncia que él mismo debe enarbolar frente a ciertos velos que la dominación social impone. Por ello, P. Bourdieu postula que la sociología debe ser una ciencia que incomoda. Y fundamentalmente debe nutrirse de un lenguaje diferente al ordinario (sentido común), que es por el que la dominación se filtra.

Frente a estos postulados, la sociología pragmática de L. Boltanski resulta menos crítica del sentido común. Para ella, los actores en situación tienen ciertas competencias, no perfectas, pero que les permiten lanzarse al ensayoerror de la acción. En ese sentido, el sociólogo debe seguir las imperfectas capacidades críticas de los actores, siempre más situadas y efectivas que las críticas que puedan enarbolarse desde el rol de observador. Los actores ya son sociólogos, arman y desarman sociedad. Sobre dichas intervenciones debe posar la mirada el investigador y, en el mejor de los casos, promover críticas situadas que resultan menos visibles para la doxa. En otras palabras, el saber sociológico resulta más influyente al elevar el nivel de publicidad de ciertos saberes situados que al tomar la 
palabra de los actores dominados.

Para concluir, es verdad que P. Bourdieu no postulaba al sociólogo como un denunciador político. Planteaba incluso que la denuncia sociológica se fortalecía en su carácter constatativo de cierta realidad (en su rigurosidad científica) más que en un nivel performativo de sus discursos (como portavoz político). Esto sirve para matizar el contraste y dar cuenta una vez más de las idas y vueltas entre ambos paradigmas.

\section{Notas}

1 Es en L'amour de l'art (Bourdieu \& Darbel, 1968) donde investiga las percepciones estéticas en Francia y descubre que la actitud estética forma parte de un ethos de clase, y que cada uno de estos habitus adquiere particularidad en relación con otros habitus de clase. Por lo tanto el habitus no es una esencia sino una relación.

2 "Para escapar a las alternativas mortales en las cuales hemos encerrado a la historia o a la sociología y que, como la oposición entre el acontecimiento y la larga duración, o, en otro orden, entre "los grandes hombres” y las fuerzas colectivas, las voluntades singulares y los determinismos estructurales, reposan todas sobre la distinción, de lo individual y lo social, identificado con lo colectivo, es suficiente observar que toda acción histórica pone en presencia dos estados de la historia (o de lo social): la historia en estado objetivado, es decir la historia acumulada a lo largo del tiempo en las cosas, máquinas, edificios, monumentos, libros, teorías, costumbres, derecho, etc., y la historia en estado incorporado, devenido habitus. Aquel que levanta su sombrero para saludar reactiva sin saberlo un signo convencional heredado de la Edad Media donde, como lo recuerda Panofsky, los hombres de armas tenían la costumbre de quitarse su casco para manifestar sus intenciones pacíficas. Esta actualización de la historia es el hecho del habitus, producto de una adquisición histórica que permite la apropiación de lo adquirido histórico" (traducción propia) (Bourdieu, 1980).

3 Será en el artículo en Condition de clase et position de classe (Bourdieu, 1966), donde nuestro autor dirá que para definir una clase social no es suficiente la posición y situación de la misma en una estructura social, esto es por las relaciones que objetivamente mantiene con otras clases, sino que los individuos que la integran mantienen relaciones simbólicas que, al expresar las diferencias de situación y posición tienden a transformarlas en relaciones simbólicas. Estas últimas transfiguran los bienes en signos, privilegiando la forma por sobre la función de los bienes.

4 La sociología pragmática recupera parte del linaje del pragmatismo en la filosofía y de la tradición de la Escuela de Chicago en la sociología. En ese sentido, retoma la preocupación por una relación de ajuste mutuo entre la acción humana y su entorno (Dewey, 2004), y la configuración del sentido social a partir de las interacciones locales en escenarios situados y en copresencia física (Goffman, 1989).

$\underline{5}$ En “Finding One's Way in Social Space: A Study based on Games” (1983), L. Boltanski \& L. Thévenot realizan tres juegos situados que les permiten reflexionar sobre cómo los actores desarrollan planes que se inscriben dentro de ciertas categorías sociales. Este estudio de sociología de la vida cotidiana indaga sobre cómo los actores se relacionan con su entorno.

$\underline{6}$ L. Boltanski \& L. Thévenot dan cuenta de la inscripción situada de su régimen de la justicia. Toda movilización de principios o dispositivos en torno a un litigio sobre la justicia/injusticia de algo se da en el marco de cierto involucramiento con una situación específica.

$\underline{7}$ En “Ce dont les gens sont capables” (1990a), L. Boltanski postula una sociología de las pruebas. Allí las personas buscan probar cosas y las cosas son probadas a través de ellas. La tarea crítica de los actores debe pasar por el mundo real de la prueba, donde aparece la "evidencia” de lo que se está denunciando.

$\underline{8}$ En Le nouvel esprit du capitalisme, L. Boltanski \& E. Chiapello (1999) se concentraron en mostrar el carácter 
histórico del modelo de la justificación. Allí muestran el carácter dinámico de las pruebas y cierta reversibilidad de las relaciones sociales. Lejos de anclarse en un modelo situacional de la acción (crítica tradicional al pragmatismo), las pruebas se van constituyendo históricamente. En este sentido, el enfoque histórico-pragmático nunca hipostasia la historia como factor de influencia causal o motivacional, sino que lo ancla a series de eventos y acciones que encadenan otras series de acciones o de eventos. En síntesis, no hay historia sin acción, sino acciones que conforman historias.

$\underline{9}$ En este libro, L. Boltanski responde a muchas de las críticas recibidas en De la justification, las cuales giran en torno a cierto idealismo por el cual las disputas sociales parecen dirimirse argumentativamente. Frente a ellas, se afirma que las pruebas de fuerza y las legítimas son dos modos de vínculo entre los actores que pueden intercalarse.

$\underline{10}$ En "Du monde social en tant que scène d'un procès" (2007), L. Boltanski \& E. Claverie muestran cómo, con el formato Affaire, las relaciones entre las personas son reversibles. A partir de un estudio sobre escándalos, los autores muestran cómo ciertas relaciones de dominación (económica, religiosa o política) pueden revertirse a partir de la creación de una "gran causa” que denuncia injusticia y suma adherentes. Con el formato de un proceso jurídico, las víctimas se transforman en denunciantes, colocando en el banquillo de acusados a los que detentaban el poder acusatorio.

11 Para un desarrollo más amplio del concepto de capital ver nuestro artículo en: http://www.cuestionessociologia.fahce.unlp.edu.ar/article/view/CSe024/8427

12 Para citar sólo algunas investigaciones: Les usages sociaux de l'automobile: concurrence pour l'espace et accidents (Boltanski, 1975); Psychosociologie d'entreprise et rééducation morale (Villette, 1976); La médicalisation de l'échec scolaire [De la pédopsychiatrie à la psychanalyse infantile] (Pinell, Zafiropoulos, 1978); L'invention du "troisième âge" (Lenoir,1979); Pratique pédagogique et classes sociales. Étude comparée de 3 écoles maternelles (Geneviève, 1979).

$\underline{13}$ En numerosas entrevistas P. Bourdieu sostendrá que los usos sociales de su teoría y su aplicación a entornos sociales diversos sólo pueden tener una definición sistemática y son creados para emplearse en una forma "sistemáticamente empírica”. Nociones como la de habitus, campo y capital pueden ser definidas, pero sólo dentro del sistema teórico y no en forma aislada.

14 En el libro Ce que parler veut dire (Bourdieu, 1982) sostiene que el orden social se mantiene en parte por la imposición de esquemas de percepción que, ajustados a las clasificaciones objetivas, reconocen ese orden, lo que implica el desconocimiento de los fundamentos de dicho orden. La política comienza según P. Bourdieu con la denuncia de ese contrato de adhesión al orden que establece la doxa ordinaria. La subversión política requiere de una subversión cognitiva que transforme la visión del mundo.

15 Entre las múltiples definiciones de sentido práctico esbozadas por P. Bourdieu, como dimensión del habitus nos interesa rescatar la siguiente: "Principio generador largamente instalado por improvisaciones reguladas, el habitus como sentido práctico opera la reactivación del sentido objetivado en las instituciones: producto del trabajo de inculcación y de apropiación que es necesario para que esos productos de la historia colectiva que son las estructuras objetivas alcancen a reproducirse bajo la forma de disposiciones duraderas y ajustadas que son la condición de su funcionamiento (...)” (Bourdieu 2007, p. 93).

16 A partir de la configuración del régimen de la justicia, postulado por L. Boltanski y L. Thévenot (1991), la mayoría de los sociólogos pragmáticos se lanzaron a reflexionar sobre qué hacen los actores cuando no se orientan a un debate público como forma de resolver disputas ordinarias o políticas. Con esa modalidad, L. Boltanski (1990) va a ser el primero en afirmar que existen cuatro modos de acción posibles (aunque no exhaustivos): el ya mencionado de la justicia, pero también el de la rutina (donde las personas articulan sus prácticas a través de reglas y dispositivos en común sin que ellas nunca se pongan en cuestión), el del amor (donde las personas se relacionan a través de la empatía y de la singularidad de uno y el otro) y el de la violencia (donde las personas 
dirimen sus conflictos a través del aniquilamiento del otro). L. Thévenot (2016) va a estructurar otra arquitectura de regímenes: desde una competencia situada y apegada al entorno (régimen familiar), pasando por una capacidad para desarrollar proyectos y estrategias (régimen del plan), hasta llegar a una competencia compleja de incorporación de ideas de bien común, de reglamentos y de retóricas oficiales (régimen público). Por último, la mayoría de los sociólogos que comenzaron a colonizar los diferentes ámbitos de la disciplina se encargaron de distinguir una arquitectura moral y a la vez realista que los actores movilizan a la hora de mostrarse competentes socialmente. C. Lemieux (1995) va a hacer referencia a un régimen de lo público ligado a los medios de comunicación; P. Corcuff (1999) va a dar cuenta de un régimen maquiaveliano a la hora de entender las prácticas políticas y administrativas; J. L. Derouet (1989) hará mención a un régimen escolar de acción para reflexionar sobre los problemas de la escuela pública; J.P Heurtin (1999) va a dar cuenta de los dispositivos y modos de acción de la actividad político-parlamentaria; F. Chateaurayaud (c/ Torny, 1999) presentará un modo de acción particular dentro de los problemas de riesgo ambiental; así como N. Dodier (2005) hará lo mismo para comprender las prácticas de los actores en el ámbito de la salud.

17 En ese artículo el autor hace referencia a los regímenes que denomina modos de acción. Afirma que son formatos de acción que las personas pueden hacer uso de manera variable y durante lapsos de tiempo muy breves para dilucidar sus relaciones (correctas o incorrectas) dentro de un contexto.

18 Allí, L. Boltanski (1990) muestra los límites del uso pragmático de los transportes de legitimidad. Cuando estos son demasiado lejanos al caso son "acusados de locura". Por ejemplo, en el caso de un problema de índole personal que se justifica a través de gramáticas públicas demasiado elevadas, como "la represión estatal” o "la explotación capitalista”. En estos casos, todo el trabajo de la justificación cae en saco roto.

19 Las investigaciones de esta compilación se realizaron en empresas (públicas o privadas) y en la administración pública (central o regional) y en escuelas.

$\underline{20}$ Los autores presentan seis formas de justificación plurales o gramáticas de bien común que funcionan como sistemas de equivalencia normativos en la mayoría de las disputas públicas, desde las más ordinarias hasta las de mayor impacto institucional o político. La primera referencia normativa se denomina cívica e invoca a la construcción de una voluntad colectiva y a la noción de igualdad ante la ley. La segunda se denomina industrial y hace referencia a los criterios de eficacia, buena planificación y competencia de los agentes. La tercera se denomina doméstica y se funda en la confianza interpersonal y la seguridad en los vínculos. La cuarta se denomina de la opinión y se basa en el reconocimiento del público del carácter notable de alguna persona. La quinta se denomina mercantil y se funda en la escasez de recursos y en las cualidades de equilibrio que otorga las relaciones económicas en el mercado. Por último, la sexta se denomina inspirada y remite a las cualidades de una persona para observar y coordinar un conjunto de relaciones sociales. Cada esquema normativo tiene una lógica propia y pone en valor imperativos de legitimidad y generalidad para la crítica y para la justificación, es decir, tanto para la denuncia como para la defensa de un orden.

$\underline{21}$ Para dar un ejemplo podríamos decir que en el imaginario de la sociedad francesa, el sistema escolar constituía la institución por excelencia del modelo de movilidad social ascendente, democrática y republicana, que contribuiría a construir un mundo social con mayores niveles de igualdad en el plano económico y en el cultural. Pero esta creencia fue desmantelada por las investigaciones llevadas adelante por P. Bourdieu y J.C. Passeron (1964; 1965; 1970) sobre el sistema educativo. Sus trabajos tuvieron un amplio reconocimiento internacional por la relativa homogeneidad con que se habían ido institucionalizando los sistemas escolares en los distintos países occidentales. Los autores brindaban una matriz que funcionó como teoría general para explicar, comprender y denunciar el papel esencialmente reproductor de las desigualdades sociales que llevaba adelante el sistema escolar en su conjunto. Lejos de asegurar libertades, igualdad y ascenso social, la Escuela contribuía, a través de la dominación cultural y simbólica, a aumentar y a legitimar volviendo aceptables diferencias sociales.

$\underline{22}$ En este párrafo hacemos referencia a la máxima pragmatista, formulada por C. Peirce (2008), la cual dice que nuestra concepción de un objeto es igual a la totalidad de las consecuencias que produce el mismo. A partir de 
ella, la verdad se desencarna de una idea por fuera de la realidad que la representa, para afirmar que la verdad de un objeto es ni más ni menos que sus consecuencias prácticas. Lo que produce el objeto es la cualidad del objeto mismo. No hay nada más allá que lo explique, por detrás de los efectos evidenciables en el mundo (Dewey, 1994; James, 2007).

$\underline{23}$ En The reality of moral expectations: a sociology of situated judgment (2000), L. Boltanski (c/ L. Thévenot) explora las formas por las cuales las personas en su vida ordinaria movilizan permanentemente órdenes de justicia para justificar sus acciones. Estos esquemas no son ideales sino que están nutridos de las reglas simbólicas y materiales de la situación.

24 En Sociologie critique et sociologie de la critique (1990b), L. Boltanski expone las diferencias entre su sociología y la de P. Bourdieu. Allí formula la distancia entre el rol crítico del investigador y los roles críticos de los actores.

$\underline{25}$ En Affaires, scandales et grandes causes. De Socrate à Pinochet (2007), L. Boltanski (c/Claverie E., Offenstadt N., Van Damme S., Eds) muestra histórica y empíricamente cómo se construyeron grandes causas que revirtieron grandes injusticias y dieron lugar a grandes críticas públicas. Allí se retoma el principio de sociología de los affaires (ya citado anteriormente), mediante el cual la disciplina debe dar cuenta de cómo las personas ordinarias revierten críticamente situaciones de injusticia o de dominación.

$\underline{26}$ En "Endless Disputes from Intimate Injuries to Public Denunciations" (1996), L. Boltanski despliega empíricamente las capacidades críticas de las personas en casos de denuncias personales.

$\underline{27}$ En De la justification (1991), L. Boltanski (c/ L. Thévenot) analiza en detalle los distintos grados de involucramiento de las personas en las situaciones, cómo en determinados momentos logran desarrollar una competencia crítica y en el mejor de los casos, cómo logran transformar la realidad que los rodea.

$\underline{28}$ En De la critique (2009), L. Boltanski desarrolla su perspectiva pragmática de una sociología de la emancipación que se orienta a la promoción de las críticas de los actores. Allí explica cómo los actores elaboran una crítica cuando se produce un desfase entre el nivel semántico y pragmático de la situación; entre las tipificaciones de las situaciones y lo que ocurre realmente.

\section{Bibliografía general}

Barthe, Y. et al. (2013). Sociologie pragmatique: mode d'emploi. Politix 26(103), 175-204.

Becker, H. (2008). Los mundos del arte. Sociología del trabajo artístico. Buenos Aires: Universidad Nacional de Quilmes Editorial.

Boltanski, L. y Chiapello E. (1999). Le nouvel esprit du capitalisme. París : Gallimard.

Boltanski, L. y Claverie E. (2007). Du monde social en tant que scène d'un procès. En Boltanski, L., Claverie E., Offenstadt N., y Van Damme S. (Eds.) Affaires, scandales et grandes causes. De Socrate à Pinochet (pp. 395-452). París: Stock.

Boltanski, L. y Thévenot, L. (1983). Finding one's way in social space. A study based on games. Social Science Information, 22(4-5), 631-680.

Boltanski, L. y Thévenot, L. (2000). The reality of moral expectations: a sociology of situated judgment. Philosophical Explorations, III(1).

Boltanski, L. y Thévenot, L. (1999). The Sociology of Critical Capacity. European Journal of Social Theory, 2(3), 359-378. 
Boltanski, L. y Thévenot, L. (1991). De la justification. Les économies de la grandeur. París : Gallimard.

Boltanski, L. y Thévenot, L. (1989). Justesse et justice dans le travail. Cahiers Du Centre d'Etudes de l'emploi. París : PUF.

Boltanski, L., Claverie E., Offenstadt N., y Van Damme S. (coord.) (2007). Affaires, scandales et grandes causes. De Socrate à Pinochet. París: Stock.

Boltanski, L. (1996). Endless Disputes from Intimate Injuries to Public Denunciations. Networks and Interpretation, Department of Sociology, Cornell University.

Boltanski, L. (1975). La constitution du champ de la bande dessinée. Actes de la Recherches en sciences Sociales, 1, 37-59.

Boltanski, L. (1978). Les cadres autodidactes. Actes de la Recherche en Sciences Sociales, 22, 3-23.

Boltanski, L. (1975). Les usages sociaux de l'automobile: concurrence pour l'espace et accidents. Actes de la Recherches en sciences sociales, 2, 25-49.

Boltanski, L. (2009). De la critique. Précis de sociologie de l'émancipation. París : Gallimard.

Boltanski, L. (1990). L'amour et la justice comme compétences. Trois essais de sociologie de l'action. París : Métaillé.

Boltanski, L. (1990a). Sociologie critique et sociologie de la critique. Politix, 10-11, 124-134.

Bourdieu, P. y Passeron, J.C. (1964). Les Héritiers, les étudiants et la culture. París: Minuit.

Bourdieu, P. y Passeron, J.C. (1965). Rapport pédagogique et communication. Cahiers du Centre de Sociologie Europeénne. París: Mouton.

Bourdieu, P. (1966). Condition de classe et position de classe. Archives Européennes de Sociologie, VII, $201-223$.

Bourdieu, P. y Passeron, J.C. (1970). La Réproduction, éléments pour une théorie du système d'enseignement. París: Minuit.

Bourdieu, P. (1990). Sociología y Cultura. México: Grijalbo.

Bourdieu, P., y Darbel, A. (1968). L’amour de l’art, Les musées d’art européens et leur public. París: EHESSMouton.

Bourdieu, P. (2006). Argelia 60, Estructuras económicas y estructuras temporales. Buenos Aires: Siglo XXI.

Bourdieu, P. (1966). Champ intellectuel et projet créateur. Les Temps Modernes, (246), 865-906.

Bourdieu, P. (1972). Esquisse d’une théorie de la pratique. Ginebra : Droz.

Bourdieu, P. (1982). Ce que parler veut dire. París: Fayard.

Bourdieu, P. (1988). La Distinción, criterios y bases sociales del gusto. Madrid: Taurus.

Bourdieu, P. (1997). Capital cultural, escuela y espacio social. Buenos Aires: Siglo XXI.

Bourdieu, P. (2007). El sentido práctico. Buenos Aires: Siglo XXI.

Bourdieu, P. (1980). Le mort saisit le vif. Les relations entre l’histoire réifiée et l'histoire incorporée. Actes de la recherche en sciences sociales, 32(1).

Bourdieu, P. (2015). Sociologie Générale Vol. I. París : Editions du Seuil. 
Chateauraynaud, F. y Torny D. (1999). Les sombres précurseurs : une sociologie pragmatique de l'alerte et du risque. París: Ecole des hautes études en sciences sociales.

Chauviré, C. y Fontaine, O. (2008). El vocabulario de Bourdieu. Buenos Aires : Atuel.

Corcuff, P. (1999). De Machiavel au régime d'action machiavélien. Philosophie Politique et Sociologie Politique. VIe Congrès de l'Association Française de Science Politique, Rennes.

Dannepond, G. (1979). Pratique pédagogique et classes sociales. Étude comparée de 3 écoles maternelles. Actes de la recherche en sciences sociales, 30, 31-45.

Derouet, J.L. (1989). L'établissement scolaire comme entreprise composite. Programme pour une sociologie des établissements scolaires. En Boltanski, L. y Thévenot, L. (Eds.), Justesse et justice dans le travail (pp. 11-42). París: PUF.

Dewey, J. (1994). La reconstrucción de la filosofía. Barcelona: Planeta.

Dewey, J. (2004). La opinión pública y sus problemas. Madrid: Ediciones Morata.

Dodier, N. (2005). O espaco e o movimento do sentido crítico. Forum Sociologico, (13-14), 239-277.

Dodier, N. (1991). Agir dans plusieurs mondes. Critique, (529-530).

Goffman, E. (1989). La presentación de la persona en la vida cotidiana. Buenos Aires: Amorrortu.

Goffman, E. (2006). Frame Analysis. Los marcos de la experiencia. Madrid: Siglo XXI.

Heurtin, J.P. (1999). L'Espace public parlementaire : essai sur les raisons du législateur. París: PUF.

James, W. (2007). Le pragmatisme. Un nouveau nom pour d’anciennes manières de penser. París: Flammarion.

Latour, B. (2001). Irréductions. París: La Découverte.

Latour, B. (2008). Reensamblar lo social. Una introducción a la teoría del actor-red. Buenos Aires: Manantial.

Leberon, F. y Mauger, G. (2012). Lectures de Bourdieu. París: Ellipses Editions.

Lemieux, C. (1995). Parler en public. Politix, 31, 5-20.

Lenoir, R. (1979). L'invention du "troisième âge". Actes de la recherche en sciences sociales, 26-27, 57-82.

Peirce, C. (2008). El pragmatismo. Madrid: Editorial Encuentro.

Pinell, P. y Zafiropoulos, M. (1978). La médicalisation de l'échec scolaire [De la pédopsychiatrie à la psychanalyse infantile]. Actes de la recherche en sciences sociales, 24, 23-49.

Schutz, A. (1987). Le chercheur et le quotidien. París: Meridiens.

Thévenot, L. (2016). La acción en plural. Una introducción a la sociología pragmática. Buenos Aires: Siglo XXI.

Villette, Michel (1976). Psychosociologie d'entreprise et rééducation morale. Actes de la recherche en sciences sociales, 2(4), 47-65. 